\title{
mTOR signaling pathway is inhibited downstream of the cyclophilin D-mediated mitochondrial permeability transition in honokiol-triggered regulated necrosis
}

\author{
WEI TIAN ${ }^{1,2}$, JIENI XIONG ${ }^{1}$, SAISA ZHU ${ }^{1}$, DONG XU ${ }^{1,2}$, HONG SHEN $^{3}$ and YONGCHUAN DENG ${ }^{1}$ \\ ${ }^{1}$ Department of Surgical Oncology; ${ }^{2}$ Cancer Institute (The Key Laboratory of Cancer Prevention and Intervention, \\ China National Ministry of Education); ${ }^{3}$ Department of Medical Oncology, The Second Affiliated Hospital, \\ School of Medicine, Zhejiang University, Hangzhou, Zhejiang 310009, P.R. China
}

Received March 19, 2015; Accepted January 20, 2016

DOI: $10.3892 / \mathrm{mmr} .2016 .4885$

\begin{abstract}
Honokiol (HNK) is a pharmacologically active small molecule that is isolated from the traditional Chinese medicinal herb, houpu. It may induce diversified types of regulated cell death, which are dependent on different cell types and varying concentrations of therapeutic agent. We previously reported that HNK triggers a cyclophilin D (CypD)-mediated regulated necrosis in various cell lines at certain concentrations (two-fold higher than its half maximal inhibitory concentration). Subsequent study revealed that HNK induced cell death transition from early apoptosis to regulated necrosis in parallel with the increase of HNK dose. In the current study, a lower concentration of $\mathrm{HNK}(30 \mu \mathrm{g} / \mathrm{ml})$ than previously reported also induced simplex CypD-mediated mitochondrial permeability transition (MPT)-associated regulated necrosis in the HEK-293 human embryonic kidney cell line. HNK, at concentration of $30 \mu \mathrm{g} / \mathrm{ml}$, induced necrotic cell death in HEK-293 cells, which was demonstrated by positive staining for propidium iodide. No DNA ladder patterns or apoptotic bodies were detected in cells that underwent this type of necrotic cell death. Caspase- 8 and -3 were not activated during the process of HNK-induced necrosis. In addition, pan-caspase inhibitor, z-VAD-fmk and receptor-interacting protein 1 inhibitor, necrostatin-1 did not inhibit HNK-induced necrosis. However, CypD inhibitor, cyclosporin A (CsA), blocked HNK-induced
\end{abstract}

Correspondence to: Dr Hong Shen, Department of Medical Oncology, The Second Affiliated Hospital, School of Medicine, Zhejiang University, 88 Jiefang Road, Hangzhou, Zhejiang 310009, P.R. China

E-mail: shenhong0023@zju.edu.cn

Professor Yongchuan Deng, Department of Surgical Oncology, The Second Affiliated Hospital, School of Medicine, Zhejiang University, 88 Jiefang Road, Hangzhou, Zhejiang 310009, P.R. China E-mail: dengyongchuanzju@gmail.com

Key words: honokiol, regulated necrosis, mammalian target of rapamycin, cyclophilin D necrosis. These findings indicate that $30 \mu \mathrm{g} / \mathrm{ml} \mathrm{HNK}$ induced simplex CypD-mediated MPT-associated regulated necrosis in HEK-293 cells. Furthermore, the findings demonstrated that during HNK-triggered regulated necrosis the mammalian target of rapamycin (mTOR) signaling pathway is also inhibited. Pretreatment with CsA, therefore, inhibits HNK-triggered regulated necrosis and reverses dephosphorylation of Akt, eIF4E-binding protein 1 and S6 kinase. This indicated that the mTOR signaling pathway is effective downstream of the CypD-mediated MPT and before the onset of plasma membrane breakdown during the regulated necrosis process. Therefore, it has been demonstrated for the first time, to the best of our knowledge, that the mTOR signaling pathway was inhibited downstream of the CypD-mediated MPT in the process of HNK-induced regulated necrosis.

\section{Introduction}

Necrosis was initially considered to be a form of accidental cell death, which only occurred in response to physicochemical injuries. Due to modern, genetic and molecular biological techniques, this concept has changed. Necrosis is now recognized to be programmed by multiple signaling pathways, and is defined as regulated necrosis in order to distinguish this genetically controlled, necrotic-like cell death process (1-3). A range of regulated necrosis initiators have been identified in recent years, including the necrosome, poly (ADP-ribose) polymerase 1 (PARP1) hyperactivation, mitochondrial permeability transition (MPT), mitochondrial complex I, nicotinamide adenine dinucleotide phosphate (NADPH)-oxidases amongst others. In addition to the well-known receptor-interacting protein 1 (RIP1) complex (the necrosome), cyclophilin D (CypD)-mediated MPT is another important initiator of regulated necrosis (2). Mammalian target of rapamycin (mTOR) is an evolutionarily conserved serine (Ser)/threonine (Thr) protein kinase. The mTOR signaling pathway promotes translation initiation and elongation, ribosome biogenesis and autophagy, which subsequently regulate cell survival and cell growth. Aberrant activation of the mTOR signaling pathway promotes cell growth and survival, particularly in malignant cells. Furthermore, although previous evidence indicates that 
limited activation of the Akt/mTOR signaling pathway is anti-apoptotic, numerous recent reports illustrate an emerging role for the Akt/mTOR signaling pathway as a death kinase and a key regulator of regulated necrosis (4).

HNK is a pharmacologically active small molecule isolated from the traditional Chinese medicinal herb, houpu. Recent evidence demonstrates that it may induce diversified types of regulated cell death, including extrinsic and intrinsic apoptosis, regulated necrosis and paraptosis, depending on varying cell types and concentration of therapeutic agents (5). It has been previously reported that HNK may trigger a CypD-mediated regulated necrosis in numerous cell lines at certain concentrations (two-fold higher than its half maximal inhibitory concentration; $\left.\mathrm{IC}_{50}\right)(6)$. The aim of the current study was to demonstrate whether HNK triggered uniform necrotic cell death in HEK-293 cells at a reduced concentration $(30 \mu \mathrm{g} / \mathrm{ml})$. In addition, the function of Akt/mTOR signaling in the process of HNK-triggered regulated necrosis was investigated.

\section{Materials and methods}

Materials and cell culture. HNK powder was purchased from the National Institute for the Control of Pharmaceutical and Biological Products (Beijing, China; $>99 \%$ purity) and was dissolved as $20 \mathrm{mg} / \mathrm{ml}$ in dimethyl sulfoxide (75 mM). RIP1 inhibitor, Nec-1, and pan-caspase inhibitor, z-VAD-fmk, were obtained from Santa Cruz Biotechnology, Inc. (Santa Cruz, CA, USA). CsA, Hoechst 33342, dimethyl sulfoxide and VP-16 were purchased from Sigma-Aldrich (St. Louis, MO, USA). the CCK-8 Cell Counting kit, DNA Ladder Detection kit and MitoTracker probes (MitoRed) were purchased from Nanjing KeyGEN Biotech Co., Ltd. (Nanjing, China). M-PER Mammalian Protein Extraction Reagent, Halt Protease and Phosphatase Inhibitor Cocktails were purchased from Thermo Fisher Scientific, Inc. (Waltham, MA, USA). The Annexin V-fluorescein isothiocyanate (FITC) Apoptosis Detection kit II was purchased from BD Biosciences (Franklin Lakes, NJ, USA). Cleaved caspase-3 and -8, B-cell lymphoma 2 (Bcl-2), RIP1, phosphorylated (p)-Akt (T308), p-Akt (S473), Akt, p-eIF4E-binding protein 1 (4E-BP1), 4E-BP1, p-S6 kinase (S6K), S6K and GAPDH antibodies were purchased from Cell Signaling Technology, Inc. (Danvers, MA, USA). Millicell EZ Slides were purchased from Merck Millipore (Darmstadt, Germany). Dulbecco's modified Eagle's medium (DMEM) and fetal bovine serum (FBS) were purchased from Gibco (Thermo Fisher Scientific, Inc.). Enhanced BCA Protein Assay Kit (P0009) was purchased from Beyotime Institute of Biotechnology (Haimen, China). Polyvinylidene difluoride (PVDF) membranes were purchased from Bio-Rad Laboratories, Inc. (Hercules, CA, USA). The HEK-293 human embryonic kidney cell line was obtained from the Cancer Institution of Zhejiang University (Hangzhou, China). The HEK-293 cells were cultured in DMEM supplemented with $10 \%$ FBS. HEK-293 cella were cultured at $7^{\circ} \mathrm{C}$ and passaged every 3-4 days.

Cell toxicity assay. HEK-293 cells were seeded in 96-well culture plates at density of 6,000 cells/per well. Following culture for $24 \mathrm{~h}$, cells were pretreated with DMEM/FBS, $25 \mu \mathrm{M}$ CsA (2 h) or $60 \mu \mathrm{M}$ Nec-1 $(1 \mathrm{~h})$. Then cells were incubated with the drug-free medium, HNK of different concentrations (30 or $40 \mu \mathrm{g} / \mathrm{ml})$ for different durations (1,2 and $4 \mathrm{~h})$. Subsequently, the CCK-8 kit was added to the culture medium at $10 \mu \mathrm{l}$ per $100 \mu \mathrm{l}$ medium. Following culture with CCK-8 for $1 \mathrm{~h}$, the optical density at a wavelength of $450 \mathrm{~nm}\left(\mathrm{OD}_{450}\right.$ ) was tested using a spectrophotometer (Model 680; Bio-Rad Laboratoeis, Inc.). The relative cell viability was calculated using the $\mathrm{OD}_{450}$ values.

Flow cytometric analysis for cell apoptosis and necrosis. HEK-293 cells were cultured in 6-cm culture dishes at density of $1.2 \times 10^{6}$ in $3 \mathrm{ml}$ medium for $24 \mathrm{~h}$. Cells were pretreated with drug-free medium, $25 \mu \mathrm{M}$ CsA $(2 \mathrm{~h}), 60 \mu \mathrm{MNec}-1$ (1 h) or $50 \mu \mathrm{M} \mathrm{z}$-VAD-fmk $(0.5 \mathrm{~h})$. The cells were treated with drug-free medium or $30 \mu \mathrm{g} / \mathrm{ml} \mathrm{HNK}$ (liquid volume, $3 \mathrm{ml}$ ) for varying durations. Following treatment, the cells were harvested and stained with Annexin V-FITC/propidium iodide (PI) and assayed by flow cytometry, as previously described $(7,8)$.

DNA ladder detection. Cells were treated as described above for flow cytometric analysis. DNA extractions from HEK-293 cells from each treatment group were performed according to the manufacturer's instructions of the DNA Ladder Detection Kit. HL-60 cells that had been treated with $20 \mu \mathrm{g} / \mathrm{ml} \mathrm{VP-16}$ for $6 \mathrm{~h}$ served as a positive control.

Chromatin condensation assessment using Hoechst 33342 staining. Cells were cultured on Millicell EZ Slides at a density of $4 \times 10^{4}$ cells/slide and treated as described above for flow cytometric analysis. Following treatment, cells were stained with Hoechst 33342 and MitoRed according to the manufacturer's instructions. The cells were examined under a confocal microscope (TCS SP5; Leica Camera, Wetzlar, Germany), as previously described (8).

Western blot analysis. Cells were treated and harvested as described above for flow cytometric analysis. Proteins were extracted using M-PER ${ }^{\circledR}$ Mammalian Protein Extraction Reagent supplemented with Halt Protease and Phosphatase Inhibitor Cocktail. Protein concentrations were determined using the BCA method. Samples (20 $\mu \mathrm{g}$ of each) were loaded in 10 or $8 \%$ sodium dodecyl sulfate polyacrylamide gel and electrophoresis was performed using PVDF membranes (100-150 V; 0.5-2 h), followed by western blotting. Images were obtained by chemiluminesence using ECL Plus Western Blotting Substrate (Thermo Fisher Scientific, Inc.). The procedures were performed as previously described (8).

Statistical analysis. SPSS version 20.0 (IBM SPSS, Amronk, NY, USA) was used to perform all statistical analyses. All the experiments were performed in triplicate, and comparable results were obtained from the three different experimental setups. The data are presented as means \pm standard deviation. Paired student's $t$-test was used in single group comparisons and $\mathrm{P}<0.01$ was considered to indicate a statistically significant difference.

\section{Results}

HNK triggers necrotic cell death in HEK-293 cells. In the current study, $30 \mu \mathrm{g} / \mathrm{ml} \mathrm{HNK}$, a lower concentration than 
A a

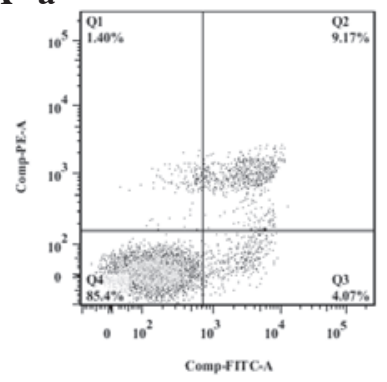

c

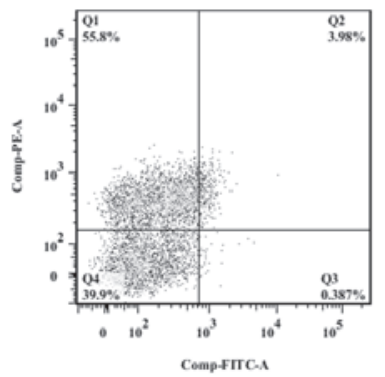

b

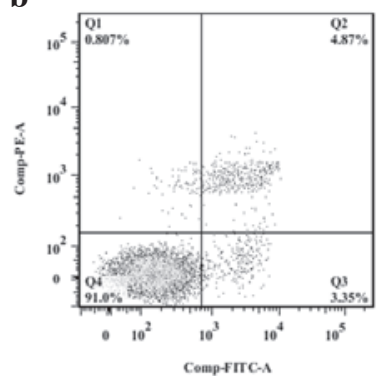

d

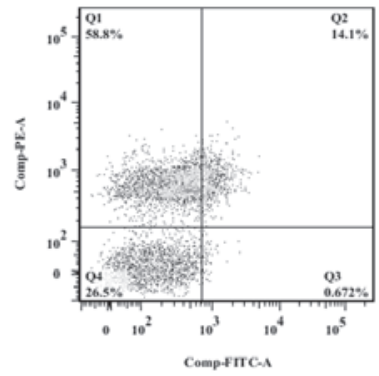

B

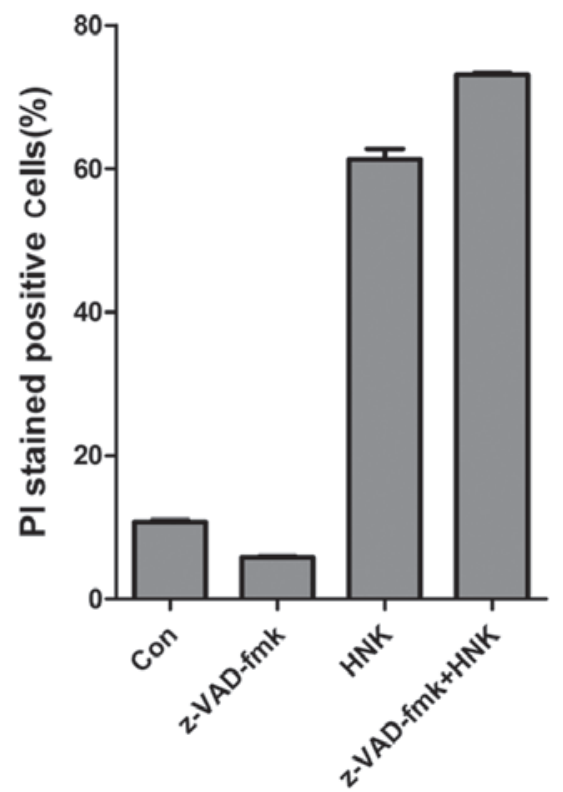

Figure 1. Pan-caspase inhibitor, z-VAD-fmk fails to reverse HNK-induced necrosis in HEK-293 cells. The HEK-293 cells were treated with drug-free medium or HNK $(30 \mu \mathrm{g} / \mathrm{ml})$ for $4 \mathrm{~h}$ in the presence or absence of pretreatment with z-VAD-fmk (50 $\mu \mathrm{M} ; 0.5 \mathrm{~h})$. Cells were labeled with Annexin V-FITC/PI and flow cytometric analysis was performed. (A) Flow cytometric analysis indicated that (a) z-VAD-fmk inhibited a portion of apoptosis compared with the (b) Con group. (c) HNK triggered a necrotic like cell death (the majority of dead cells were stained by PI). (d) z-VAD-fmk did not inhibit HNK-induced necrotic cell death. (B) Ratios of PI-stained positive cells in the four groups were analyzed. Results are representative of three independent experiments. The values are presented as the mean \pm standard deviation. Q1, $\mathrm{AV}(-) \mathrm{PI}(+)$; Q2, $\mathrm{AV}(+) \mathrm{PI}(+)$; Q3, AV(+)PI(-); Q4, AV(-)PI(-); HNK, honokiol; FITC, fluorescein isothiocyanate; PI, propidium iodide; Con, control.

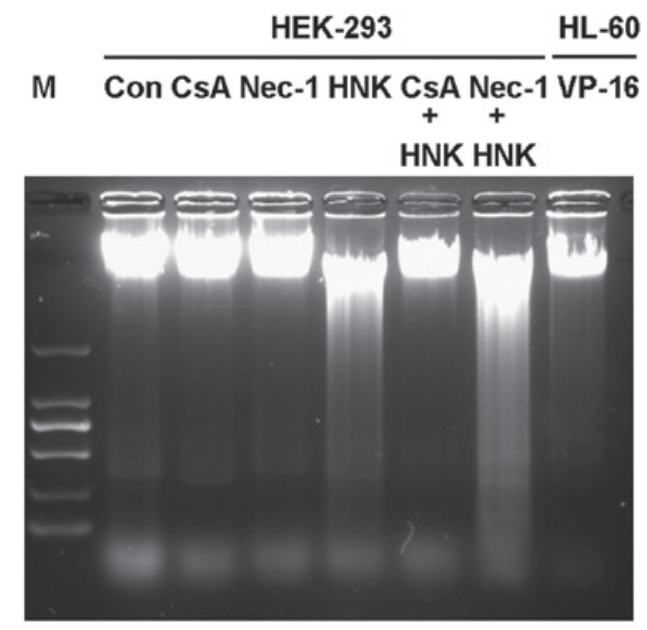

Figure 2. HNK triggers DNA diffuse fracture in HEK-293 cells, which was reversed by CsA pretreatment. Following exposure to HNK $(30 \mu \mathrm{g} / \mathrm{ml})$ for $4 \mathrm{~h}$, DNA from HEK-293 cells underwent diffuse fracture, which indicated that the majority of HNK-induced cell death resulted from necrosis. Furthermore, the HNK-triggered DNA diffuse fracture was reversed by pretreatment with CsA $(25 \mu \mathrm{M})$ for $2 \mathrm{~h}$. Nec-1 $(50 \mu \mathrm{M})$ pretreatment for $1 \mathrm{~h}$ did not inhibit HNK-triggered DNA diffuse fracture. During DNA ladder detection, HL-60 cells treated with VP-16 for $6 \mathrm{~h}$ served as a positive Con Results are representative of three independent experiments. HNK, honokiol; CsA, cyclosporin A; NEC-1, necrostatin-1.

previously reported (40 $\mu \mathrm{g} / \mathrm{ml})(6)$, was used to demonstrate its effect on HEK-293 cells. HEK-293 cells were incubated with $30 \mu \mathrm{g} / \mathrm{ml} \mathrm{HNK}$ for $4 \mathrm{~h}$, and labeled with Annexin V-FITC and PI (Fig. 1). Cell viability was significantly reduced following treatment with $30 \mu \mathrm{g} / \mathrm{ml} \mathrm{HNK}$. And the majority of HNK-triggered cell deaths were represented by positive staining for PI, which indicated necrotic cell death (Fig. 1Ac and B). Subsequently, whether the PI-stained cells had undergone necrosis or were composed of necrotic cells and undergoing advance-stage apoptosis was investigated. Internucleosomal DNA fragmentation is a morphological characteristic of apoptosis at the advanced stage (or degradation phase), which can be detected as a ladder pattern by electrophoresis of isolated DNA. Apoptotic bodies are another morphological characteristic of apoptosis and are detectable using nuclei staining with Hoechst 33342. DNA laddering and apoptotic bodies are frequently used to determine the existence of apoptosis. DNA laddering was performed on DNA extracted from HEK-293 cells after exposure to $30 \mu \mathrm{g} / \mathrm{ml} \mathrm{HNK}$. No obvious ladder pattern was observed, however a diffuse fractured pattern was detected following HNK exposure (Fig. 2). Confocal imaging of Hoechst 33342-stained nuclei was used to determine apoptotic bodies during the process of HNK-triggered necrotic cell death. Confocal imaging did not detect any nuclear chromatin condensation or apoptotic bodies in the HEK-293 cells after exposure to $30 \mu \mathrm{g} / \mathrm{ml} \mathrm{HNK}$ for $0.5,1$ or $2 \mathrm{~h}$ (Fig. 3). In addition, DNA laddering and Hoechst 33342 staining revealed no morphological manifestation of apoptosis in HNK-triggered cell death of HEK-293 cells. Caspase-8 is an important initiator caspase, and it is activated during the initiation phase of extrinsic apoptosis. Activated caspase- 8 leads to the release of its active fragments, p18 and p10. It cleaves and activates downstream effector caspases (including caspase-3, -6 , and -7 ), which in turn execute apoptosis. The present study 


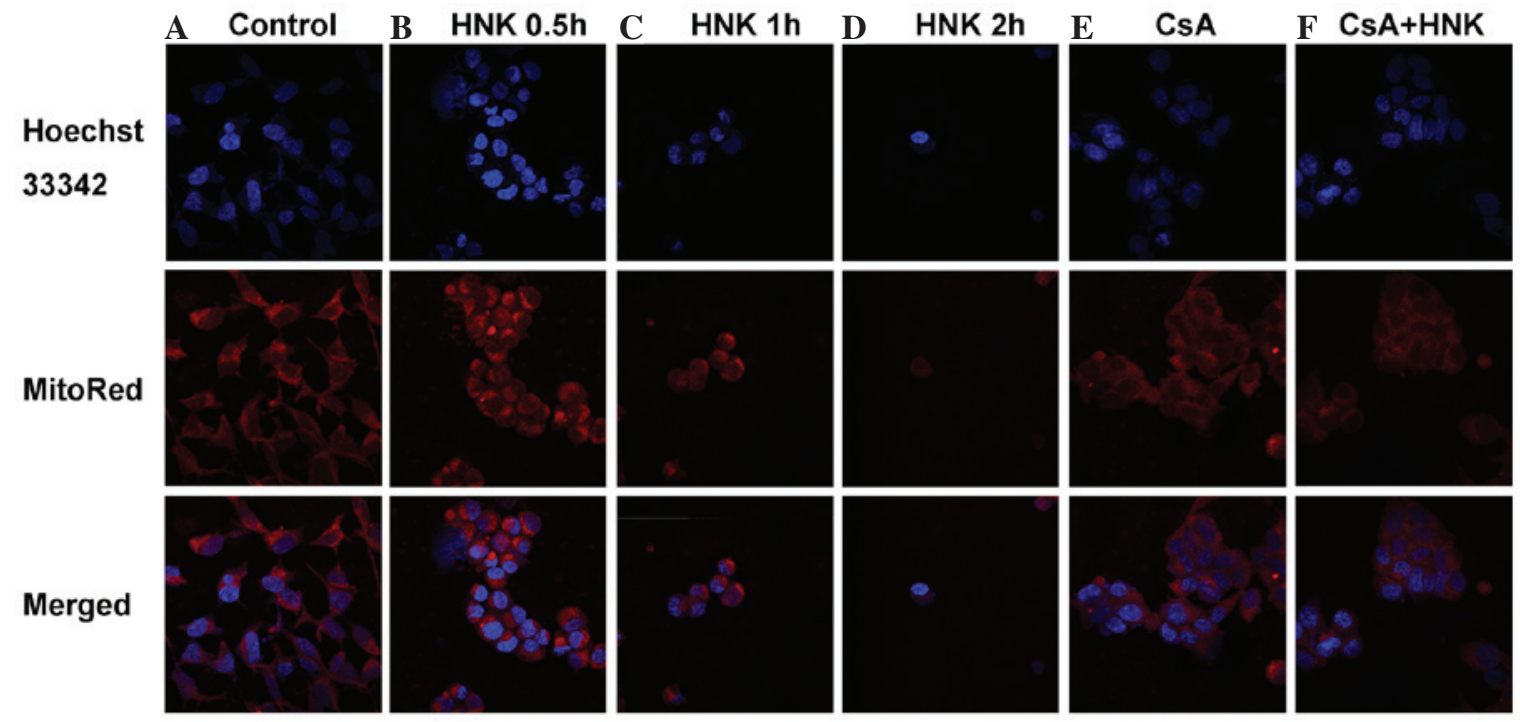

Figure 3. No chromatin condensation was detected during the process of HNK-triggered regulated necrosis. HEK-293 cells were (A) untreated (Control) or treated with $\mathrm{HNK}$ at $30 \mu \mathrm{g} / \mathrm{ml}$ for (B) 0.5 , (C) 1 and (D) $2 \mathrm{~h}$. Cells were stained with Hoechest 33342 and MitoRed, and observed under a confocal microscope. No obvious apoptotic bodies were detected during the process of HNK-triggered regulated necrosis. Mitochondrial staining was intense in the early stage of HNK-induced necrosis $(0.5 \mathrm{~h}$ ), and gradually weakened during the necrotic process ( 1 and $2 \mathrm{~h}$ ). (E and F) Signal changes of mitochondrial staining triggered by HNK were reversed by pretreatment with $25 \mu \mathrm{M} \mathrm{CsA}$. Results are representative of three independent experiments. Scale bar $=50 \mu \mathrm{m}$. HNK, honokiol; CsA, cyclosporin A.

A

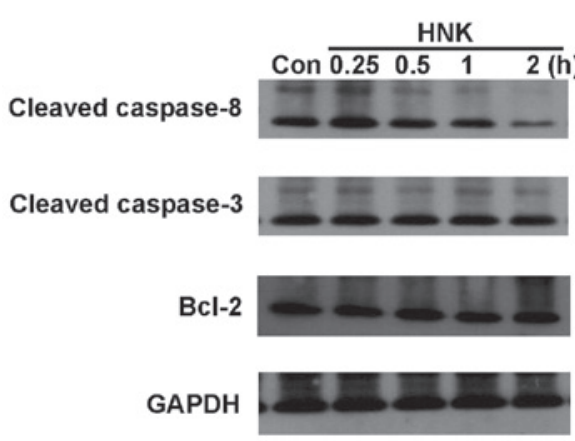

B

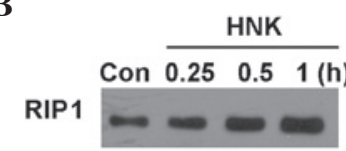

GAPDH

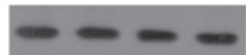

Figure 4. RIP1 expression levels increased in the ultra early stage of HNK-induced necrosis. During the process of HNK-induced regulated necrosis, it was observed that (A) cleaved caspase- 8 levels were increased in the ultra early stage and subsequently decreased in parallel with the increase of necrosis. Furthermore, protein levels of cleaved caspase-3 and Bcl-2 had not elevated though the process of HNK-triggered cell death (0.25, 0.5 , 1 and 2 h). (B) RIP1 expressions apparently increased in parallel with the increase in necrosis at the ultra early stage $(0.25,0.5$ and $1 \mathrm{~h})$. Results are representative of three independent experiments. HNK, honokiol; Con, control; Bcl-2, B-cell lymphoma 2; RIP1, receptor-interacting protein 1.

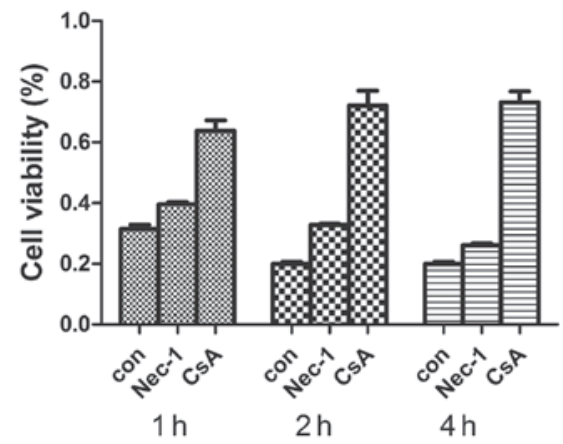

Figure 5. CsA pretreatment reverses HNK-triggered regulated necrosis regardless of treatment duration. CsA $(25 \mu \mathrm{M})$ blocked HNK-induced cell death resulting from 1-, 2- or 4-h HNK treatment. Nec-1 partially reversed HNK-induced cell death. However, the inhibition effect of Nec-1 on HNK-induced regulated necrosis decreased with the increase in HNK treatment duration. Results are representative of three independent experiments. The values are presented as the mean \pm standard deviation. ${ }^{*} \mathrm{P}<0.001$ vs. Con group. CsA, cyclosporin A; HNK, honokiol; Nec-1, necrostatin-1. demonstrated that in the ultra early stage of HNK-induced necrosis $(0.25 \mathrm{~h})$, the cleaved caspase- 8 level was increased. Subsequently, its expression level decreased in parallel with the increase in necrosis. Caspase-3 is a critical activator of intrinsic and extrinsic apoptosis. Activation of caspase-3 requires proteolytic processing of its inactive zymogen into activated p17 and p12 fragments. And active level of cleaved caspase- 3 is frequently used as a chemical manifestation to determine apoptosis. Western blot analysis revealed that the protein levels of cleaved caspase- 3 had not elevated though the process of HNK-triggered cell death $(0.25,0.5,1$ and $2 \mathrm{~h}$; Fig. 4). Bcl-2 is a pro-survival protein of the Bcl-2 family. It exerts a survival function in response to a wide range of apoptotic stimuli via inhibition of mitochondrial cytochrome $\mathrm{C}$ release. In the process of HNK-triggered cell death, no obvious changes in expression levels of $\mathrm{Bcl}-2$ were detected with increasing treatment durations using western blotting (Fig. 4). 
A
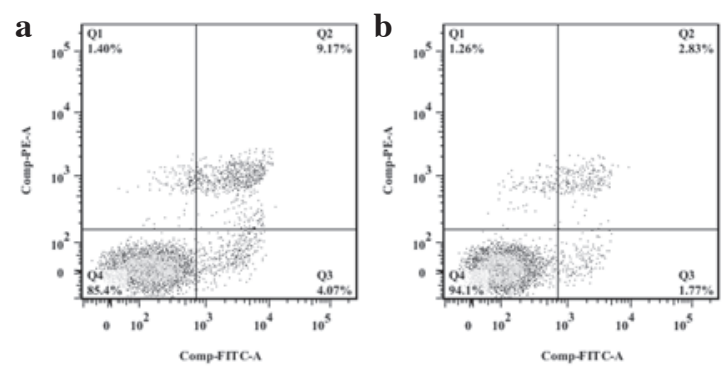

c

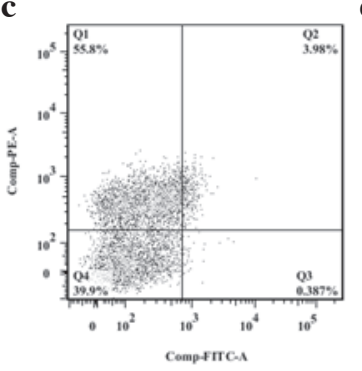

d

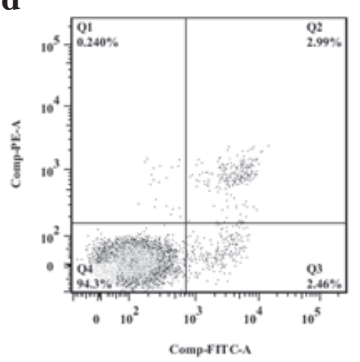

B

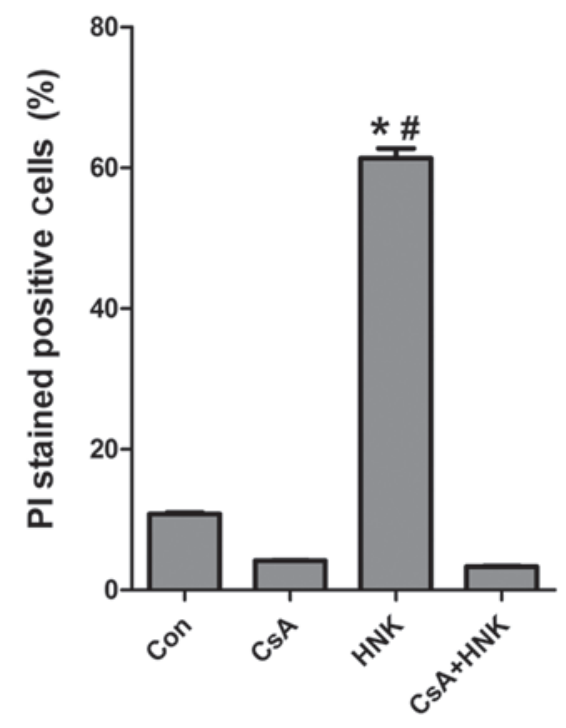

Figure 6. CsA pretreatment markedly inhibited HNK-triggered regulated necrosis. HEK-293 cells were treated with drug-free medium or HNK (30 $\mu$ g/ml) for $4 \mathrm{~h}$ in the presence or absence of CsA pretreatment $(25 \mu \mathrm{M} ; 2 \mathrm{~h})$. Cells were labeled with Annexin V-FITC and PI, and flow cytometric analysis was performed. (A) CsA pretreatment markedly reversed HNK-triggered necrotic cell death: (a) Con; (b) CsA; (c) HNK; (d) CsA + HNK. (B) Analysis of ratios of PI-stained cells in the four groups. Results are representative of three independent experiments. The values are presented as the mean \pm standard deviation. ${ }^{\sharp} \mathrm{P}<0.001$ vs. Con group; *P<0.001 vs. CsA + HNK group. Q1, AV(-)PI(+); Q2, AV(+)PI(+); Q3, AV(+)PI(-); Q4, AV(-)PI(-); CsA, cyclosporin A; HNK, honokiol; FITC, fluorescein isothiocyanate; PI, propidium iodide; Con, control.

Conversely, pan-caspase inhibitor, z-VAD-fmk did not reverse HNK-induced cell death (Fig. 1), which also demonstrated that HNK-induced cell death was caspase-independent and did not result from apoptosis. Therefore, the morphological and chemical manifestations reveal that apoptosis was not induced in HEK-293 cells by HNK at a dose of $30 \mu \mathrm{g} / \mathrm{ml}$. However, $30 \mu \mathrm{g} / \mathrm{ml} \mathrm{HNK}$ did trigger simple necrotic cell death in HEK-293 cells.

CsA blocks HNK-triggered regulated necrotic cell death. Regulated necrosis is defined as a genetically controlled cell death process, which is morphologically characterized by cytoplasmic granulation, and organelle and/or cellular swelling (2). Previous reports demonstrated that morphological manifestations of HNK-induced regulated necrotic cell death included increased cell volume (due to swelling of cytoplasmic organelles), dilatation of mitochondria and the endoplasmic reticulum, vacuoles and nuclear modifications $(6,7)$. Among the morphological manifestations (vacuoles presenting as autophagic vacuoles containing a ruptured plasma membrane, manifesting as membranous whorls), mitochondrial dilatation was the most characteristic. MitoRed was used to stain the mitochondria of HEK-293 cells, and confocal imaging was performed to detect morphological changes of the mitochondria. Intense staining of the mitochondria was observed in the early stage of HNK-induced necrosis, and gradually weakened during the necrotic process (Fig. 3). This indicated that the mitochondria underwent swelling and subsequently disintegration during $\mathrm{HNK}$-induced necrosis from the early to late stages. MPT is an initiator of the schematic steps of regulated necrosis (2). It was previously reported that $\mathrm{CypD}$, a vital protein involved in MPT, was a critical regulator in
HNK-induced regulated necrotic cell death (6-8). CypD levels gradually increased in parallel with HNK-triggered regulated necrosis. Furthermore, CypD inhibitors (CsA) or CypD siRNA depletion completely blocked $\mathrm{CypD}$, which significantly attenuated HNK-induced necrotic cell death $(6,8)$. In the current study, $25 \mu \mathrm{M}$ CsA pretreatment prior to HNK treatment was performed to determine whether CsA pretreatment inhibits HNK-induced necrosis. The results indicated that $25 \mu \mathrm{M} \mathrm{CsA}$ pretreatment for $2 \mathrm{~h}$ significantly increased cell viabilities following HNK treatment for 1, 2 and $4 \mathrm{~h}$ (Fig. 5). In addition, it completely blocked HNK-induced necrotic cell death, which returned the levels of PI-stained cell fractions to the control level. The PI-stained cell fractions in the control, CsA, HNK and CsA + HNK groups were 10.79 $\pm 0.19,4.16 \pm 0.07$, $61.33 \pm 1.42$ and $3.33 \pm 0.09 \%$, respectively ( $<<0.001$; Fig. 6). Conversely, $25 \mu \mathrm{M}$ CsA pretreatment blocked DNA diffuse fracture (Fig. 2) and mitochondrial dilatation (Fig. 3). The results indicated that $\mathrm{HNK}$-triggered regulated necrotic cell death could be completely blocked by $\mathrm{CsA}$, which indirectly indicates that $\mathrm{CypD}$ was a central initiator during the process of HNK-induced regulated necrosis in HEK-293 cells.

Necrostatin-1 (Nec-1) partly alleviates HNK-induced necrosis at the early stage. The most characteristic form of regulated necrosis is termed necroptosis. In human cells, the RIP1-RIP3-mixed lineage kinase domain-like (MLKL)-PGAM family member 5, Ser/Thr protein phosphatase, mitochondrial (PGAM5)-dynamin-related protein 1 (Drp1) axis drives tumor necrosis factor (TNF)-induced necroptosis via mitochondrial fission (9). Necroptosis is characterized as RIP1-dependent and can be specifically inhibited by RIP1 inhibitor, Nec-1. In previous studies, RIP1 
A a

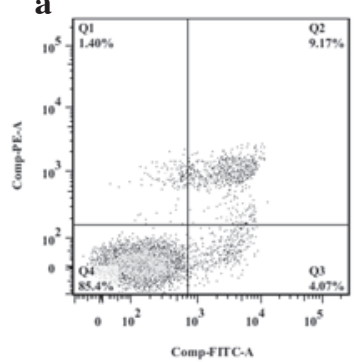

$\mathbf{C}$

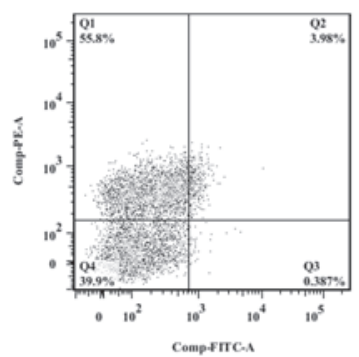

b

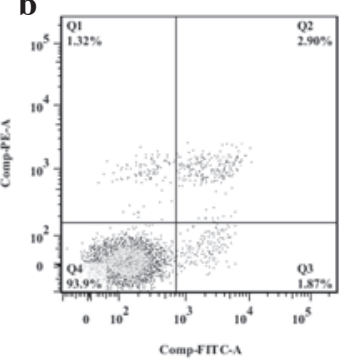

d

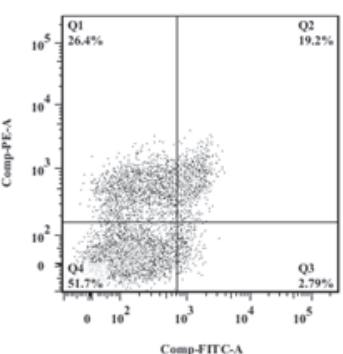

B

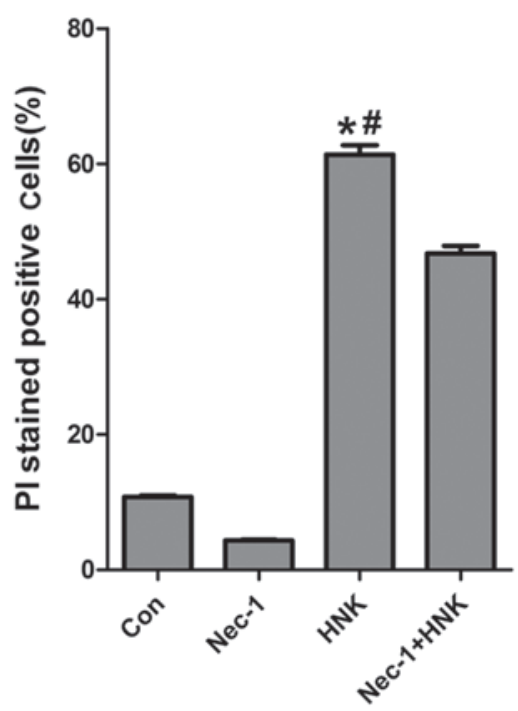

Figure 7. (A) Nec-1 partially reversed the HNK-induced regulated necrosis. (a) Con; (b) Nec-1; (c) HNK; (d) Nec-1 + HNK. (B) Results are representative of three independent experiments. The values are presented as the mean \pm standard deviation. ${ }^{*} \mathrm{P}<0.001$ vs. Con group; ${ }^{*} \mathrm{P}<0.001$ vs. Nec-1 group. Q1, AV(-)PI(+); Q2, AV(+)PI(+); Q3, AV(+)PI(-); Q4, AV(-)PI(-); NEC-1, necrostatin-1; FITC, fluorescein isothiocyanate; Con, control; HNK, honokiol.

expression levels were demonstrated to be downregulated in HNK-induced regulated necrosis in the MCF-7 breast cancer cell line throughout treatment durations of 1-4 h (8). In the current study, RIP1 expression levels and functions were detected at the ultra early stage in HNK-induced regulated necrosis in HEK-293 cells. Contrary to our previous report, RIP1 expression levels were observed to be upregulated in the ultra early stage (0.25-1 h) of regulated necrosis (Fig. 4). Subsequent results revealed that HNK-induced cell death was partly reversed by pretreatment with $60 \mu \mathrm{M} \mathrm{Nec}-1$ for $1 \mathrm{~h}$ [PI-stained cell fractions in the control, Nec-1, HNK and Nec-1 + HNK groups were 10.79 $\pm 0.19,4.36 \pm 0.12,61.33 \pm 1.42$ and $46.73 \pm 1.15 \%$, respectively $(\mathrm{P}<0.001$; Fig. 7$)]$. In addition, the inhibition effects of Nec-1 on HNK-induced regulated necrosis gradually reduced with the elongation of HNK incubating time (1-4 h; Fig. 5). The results indicated that RIP1 probably participated in the initiation stage of HNK-induced regulated necrosis. However, it was not a pivotal initiator and its effects in the process of HNK-induced regulated necrosis were limited.

mTOR signaling pathways are inhibited during HNK-triggered regulated necrosis. mTOR is a highly conserved Ser/Thr kinase, which integrates diverse signals to control cell growth, proliferation, survival and metabolism. mTOR assembles into two functionally different complexes, mTORC1 and mTORC2, with distinct inputs and downstream effects. mTORC1 regulates cell growth by promoting translation, ribosome biogenesis and autophagy via phosphorylation of its substrates, including 4E-BP1 and ribosomal S6K. mTORC2 promotes cell cycle entry, cell survival, actin cytoskeleton polarization and anabolic output though phosphorylation of its substrates, the Ser/Thr protein kinases, Akt, protein kinase A (PKA) and PKC $(10,11)$. Previous findings demonstrate that aberrant activation of the mTOR signaling pathway is vital in tumorigenesis, and promotes growth and survival of various types of cancer cells (12). Due to its high biological relevance to cancer, different therapeutic strategies have been developed to target this signaling cascade, such as mTOR inhibitors Everolimus and Temsirolimus (13). In the current study, the activation levels of the mTOR signaling pathway during HNK-triggered regulated necrosis in HEK-293 cells was determined. Western blot analysis was performed to determine the phosphorylation and expression levels of core mTOR substrates, Akt, 4E-BP1 and S6K. The phosphorylation levels of 4E-BP1 and S6K were observed to gradually decline with the elongation of incubating duration $(0.25,0.5,1$ and $2 \mathrm{~h}$ ) during the process of HNK-triggered regulated necrosis. Phosphorylation of Akt on Thr308 and Ser473 were also gradually downregulated. Conversely, the protein expression levels of Akt, 4E-BP1 and S6K were stable (Fig. 8A). Figs. 5 and 6 demonstrate that CsA markedly inhibited HNK-induced regulated necrosis. Therefore whether pretreatment with CsA could reverse dephosphorylation of mTOR substrates was investigated. Pretreatment with CsA prominently reversed dephosphorylation of Akt on Ser473, however, only partially reversed dephosphorylation of $4 \mathrm{E}-\mathrm{BP} 1, \mathrm{~S} 6 \mathrm{~K}$ and Akt on Thr308 (Fig. 8B). Collectively, the above results reveal that the mTOR signaling pathways were inhibited during the process of HNK-triggered regulated necrosis in HEK-293 cells. Furthermore, CypD may act downstream of mTOR signaling pathways, as CsA blocked HNK-triggered regulated necrosis, although it only partially reversed the dephosphorylation of mTOR substrates.

\section{Discussion}

HNK is a pharmacologically active small molecule, which is isolated from the traditional Chinese medicinal herb, houpu. A previous study evidenced its antitumor effects (5). 
A

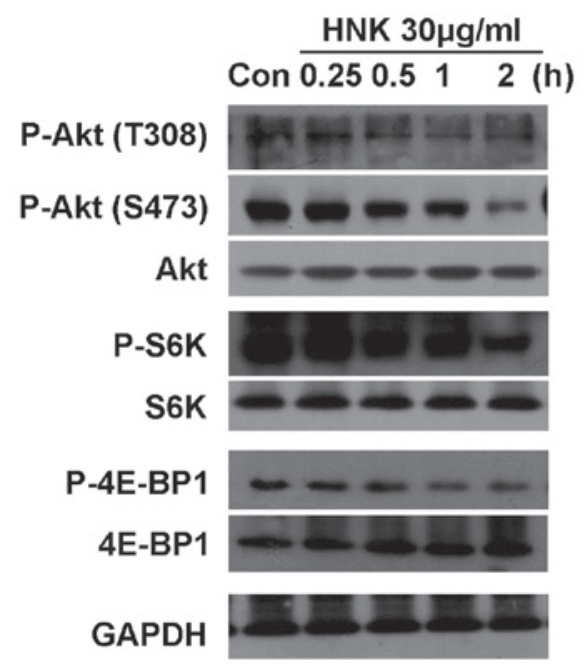

B

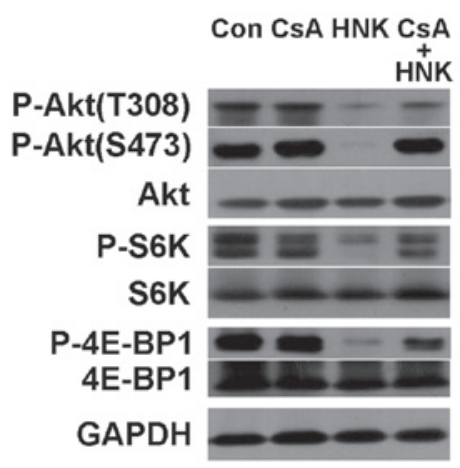

Figure 8. CsA blocks the inhibition of mTOR signaling pathways during the process of HNK-triggered regulated necrosis. (A) Phosphorylation levels of core mTOR substrates, Akt (S473 and T308), 4E-BP1 and S6K were all downregulated, whereas the expression levels of these proteins remained unchanged. (B) CsA observably blocks inhibition of the phosphorylation levels of the core mTOR substrates. Results are representative of three independent experiments. HNK, honokiol; Con, control; CsA, cyclosporin A; S6K, S6 kinases; 4E-BP1, eIF4E-binding protein 1; T, threonine; S. serine.

The antitumor function of HNK that has been investigated most is cell death induction (5). HNK may induce diverse types of regulated cell death, including extrinsic apoptosis, caspase-dependent and -independent intrinsic apoptosis, regulated necrosis and paraptosis depending on the cell type and varying concentrations of therapeutic agents.

Necrosis was initially considered as a form of accidental cell death, which only occurred in response to physicochemical insults (2). However, recent genetic and molecular biological evidence reveals that necrosis is regulated by multiple signaling pathways (2). The term regulated necrosis is defined as a genetically controlled cell death process, which is morphologically characterized by cytoplasmic granulation, and organelle and/or cellular swelling (2). A variety of regulated necrosis initiators have been identified since the introduction of this concept. Initiators of regulated necrosis include the necrosome, PARP1 hyperactivation, MPT, mitochondrial complex I, NADPH oxidases amongst others (2). Furthermore, regulated necrosis may result from CypD-mediated MPT (14). Previous studies have demonstrated HNK triggered regulated necrotic cell death in various cell lines (MCF-7, HL-60 and HEK-293) at certain concentrations (two-fold higher than its $\mathrm{IC}_{50}$ ), and this HNK-triggered regulated necrosis was demonstrated to be CypD-mediated MPT pore dependent (6). CypD inhibitors (CsA) or CypD siRNA depletion completely blocked CypD, which significantly attenuated $\mathrm{HNK}$-induced regulated necrosis. Subsequent investigations revealed HNK triggered a cell death mode transition from early stage apoptosis to regulated necrosis in a time- and dose-dependent manner, and this cell death mode transition was also highly regulated by CypD (8). In the current study, $30 \mu \mathrm{g} / \mathrm{ml} \mathrm{HNK}$, a lower concentration than a previously reported necrotic-induction concentration of $40 \mu \mathrm{g} / \mathrm{ml}$, was used (6) to demonstrate whether HNK is able to trigger a similar type of regulated necrosis in HEK-293 cells. The results of the present study demonstrated that the majority of HEK-293 cells underwent cell death, characterized by positive staining for PI, which suggested these cells lost their membrane integrity and underwent necrosis (PI-positive) or late-stage apoptosis (Annexin V-positive and PI-positive). Subsequently, morphological and biochemical methods were used to distinguish late-stage apoptosis from HNK-induced cell death. Chromatin condensation and chromosomal DNA fragmentation are two significant morphological changes that are observed in late-stage apoptosis. In the present study, DNA laddering demonstrated a diffuse DNA fractured pattern following HNK exposure, rather than DNA laddering fragmentation (2). In addition, nuclear chromatin condensation and apoptotic bodies were not detected in HEK-293 cells following HNK exposure. These preliminary morphological assessments confirmed that the HNK-induced cell death (indicated by positive staining for PI) was not due to late-stage apoptosis. Conversely, initiator caspases (caspase-8) and effector caspases (caspase-3) were not observed to be activated during this process of cell death. Pan-caspase inhibitor, z-VAD-fmk also failed to inhibit HNK-induced cell death. These results indicate that $30 \mu \mathrm{g} / \mathrm{ml} \mathrm{HNK}$-induced cell death was caspase-independent. The above-mentioned findings indicate that HNK-induced cell death was not due to late-stage apoptosis, but was solely necrosis. Subsequently, it was detected that pretreatment with CsA completely blocked HNK-induced necrosis. DNA diffuse fragmentation and mitochondrial morphological changes were reversed by CsA. These results indicated that CypD-mediated MPT was an important initiator in HNK-triggered regulated necrosis, which was previously reported $(6,8)$. Comparable to its effects in other disease models, such as ischemia-reperfusion injury (14-18), the CypD-mediated MPT-associated signaling pathway was particularly important in $\mathrm{HNK}$-induced regulated necrosis. In addition to the CypD-mediated MPT-associated signaling pathway, the RIPK1-RIPK3-MLKL-PGAM5-Drp1 axis is the most intensively investigated signaling pathway associated with a classic type of regulated necrosis, termed necroptosis. Current evidence demonstrates that necroptosis is initiated by RIP1 phosphorylation, which activates RIP3 and subsequently 
phosphorylates the MLKL protein $(1,3,19)$. Additionally, necroptosis requires the activity of RIP1, which was indicated by the protection conveyed by the RIP1 kinase inhibitor, Nec-1. In the current study, the aim was to demonstrate whether RIP1 exerted a marked effect in HNK-induced regulated necrosis in HEK-293 cells. Inconsistent with our previous report (8), RIP1 expression levels were upregulated in the ultra early stage $(0.25-1 \mathrm{~h})$ of regulated necrosis. Subsequent results revealed that although HNK-induced cell death was partly reversed by pretreatment with Nec-1, the inhibition effects of Nec-1 gradually receded with the elongation of HNK incubating time. Conversely, Nec-1 did not reverse HNK-induced DNA diffuse fragmentation. These results indicate that although the expression levels of RIP1 were upregulated in the ultra early stage of regulated necrosis, HNK-induced regulated necrosis was RIP1-independent. Collectively, these results strongly indicate that CypD-mediated MPT, but not the RIP1-associated complex, was a vital initiator in HNK-triggered regulated necrosis. However, the downstream signaling pathways of CypD-mediated MPT in HNK-triggered regulated necrosis remain unknown.

mTOR is an evolutionarily conserved Ser/Thr protein kinase, which controls cell growth in response to energy, nutrients, growth factors and other environmental precipitating factors. The deregulated mTOR signaling pathway presents in numerous types of human disease with altered metabolism, including diabetes and cancer (20). mTOR assembles into two functionally and structurally distinct complexes, mTORC1 and mTORC2 $(10,20)$. mTORC1 activity is though phosphorylation and activation of $S 6 \mathrm{~K}$, or phosphorylation and deactivation 4E-BP1, which promotes translation initiation and elongation, ribosome biogenesis and autophagy, and subsequently regulates cell growth (21). mTORC2 promotes cell cycle entry, cell survival, actin cytoskeleton polarization and anabolic output though phosphorylation of its substrates, the Ser/Thr protein kinases, Akt, PKA and PKC 10,11). Aberrant activation of the mTOR signaling pathway promotes cell growth and survival, particularly in malignant cells (12). Previous reports demonstrate that HNK downregulates expression levels and phosphorylation of Akt, S6K, and 4E-BP1 during the process of HNK-induced apoptosis (22-25). Anti-apoptotic activity of the Akt/mTOR signaling pathway via numerous mechanisms, including inhibition of caspase-9, BCL2 associated agonist of cell death and glycogen synthase kinase $3 \beta$ (GSK-3 $\beta$ ), and induction of mitochondrial hexokinase and nuclear factor $\kappa$ B-dependent anti-apoptotic gene expression, has been well documented (4). The present study aimed to investigate whether CypD-mediated MPT exerts its effect by inhibiting mTOR signaling pathways. Phosphorylation levels of mTORC1 and mTORC2 substrates (including S6K, 4E-BP1 and $\mathrm{Akt}$ ) were observed to be downregulated. Conversely, the protein expression levels of these substrates were identified to be stable, which revealed that the transcription and translation processes of these proteins had not been influenced. These results indicate that the mTOR signaling pathway was inhibited by dephosphorylation of core substrates during the process of HNK-triggered regulated necrosis in HEK-293 cells. Furthermore, these results are consistent with previous reports that HNK inhibits Akt/mTOR signaling that is accompanied by cell apoptosis. To the best of our knowledge, the current study is the first to report that the regulation of $\mathrm{Akt} / \mathrm{mTOR}$ signaling is analogous with HNK-triggered regulated necrosis. There is evidence to indicate that limited activation of Akt is anti-apoptotic, however, numerous recent reports illustrate an emerging role for Akt as a death kinase and a key regulator of programmed necrosis (4). Sustained Akt activity promotes programmed necrosis via a number of distinct mechanisms, such as oxygen consumption, enhanced ROS generation (26) and upregulation of antioxidant defenses via phosphorylation of forkhead box subclass $\mathrm{O}$ transcription factors (27). Liu et al (4) recently demonstrated that Akt and mTOR mediate TNF $\alpha / z$-VAD-fmk-induced regulated necrosis in neurons. The study revealed that RIP1-RIP3-pAkt assembly is a vital node in TNF $\alpha / \mathrm{z}-\mathrm{VAD}$-fmk-induced regulated necrosis. Activation of Akt/mTOR signaling pathways, including phosphorylation of Akt (T308 and S473), GSK-3 $\beta$, mTOR and S6K, participate in the initiation of $\mathrm{TNF} \alpha / \mathrm{z}-\mathrm{VAD}$-fmk-induced regulated necrosis in neurons. Furthermore, inhibition of Akt/mTOR signaling pathways, via a specific inhibitor or siRNA knockdown of Akt and mTOR, halves TNF $\alpha / z-V A D$-fmk-induced regulated necrosis. The study concluded that Akt and mTOR participate in regulation of TNF $\alpha / z-V A D-f m k$-induced regulated necrosis in neurons (4). These findings were not consistent with those of the present study. The discordant effects of Akt and mTOR may depend on the exact stimulus used to initiate necroptosis in unique cell types. Specifically, the functions of Akt and mTOR in CypD-mediated MPT-initiated regulated necrosis and the RIP1-RIP3 complex-initiated regulated necrosis may differ. Furthermore, pretreatment with CsA was found to prominently inhibit HNK-triggered regulated necrosis, as well as reverse dephosphorylation of Akt in Ser473/Thr308, 4E-BP1 and S6K. This indicates that the mTOR signaling pathway was effective downstream of the CypD-mediated MPT and prior to the onset of plasma membrane breakdown in the HNK-induced regulated necrosis process. Although it is known that the mTOR signaling pathway is downstream of CypD-mediated MPT, the exact signaling pathway that exists between them remains unknown. The aim of future studies is to identify the exact mechanisms between CypD-mediated MPT and inhibition of the downstream mTOR signaling pathway.

In conclusion, it was found that CypD-mediated MPT, but not the RIP1-associated complex, is a vital initiator in HNK-triggered regulated necrosis. The present study demonstrated for the first time, to the best of our knowledge, that the mTOR signaling pathway is inhibited and exists downstream of the CypD-mediated MPT in the HNK-induced regulated necrosis process. These results provide novel insight into CypD-mediated, HNK-triggered regulated necrosis.

\section{Acknowledgements}

The present study was supported by grants from Zhejiang Provincial Natural Science Foundation of China (grant nos. LQ14H160010, LY14H160019 and LY14H160020), the National Natural Science Foundation of China (grant nos. 81201640) and the Department of Education of Zhejiang Province of China (grant nos. Y201225802). 


\section{References}

1. Moujalled DM, Cook WD, Murphy JM and Vaux DL: Necroptosis induced by RIPK3 requires MLKL but not Drp1. Cell Death Dis 5: e1086, 2014.

2. Vanden Berghe T, Linkermann A, Jouan-Lanhouet S, Walczak H and Vandenabeele P: Regulated necrosis: The expanding network of non-apoptotic cell death pathways. Nat Rev Mol Cell Biol 15: $135-147,2014$.

3. Zhang J and Chan FK: Cell biology. RIPK3 takes another deadly turn. Science 343: 1322-1323, 2014.

4. Liu Q, Qiu J, Liang M, Golinski J, van Leyen K, Jung JE, You Z, Lo EH, Degterev A and Whalen MJ: Akt and mTOR mediate programmed necrosis in neurons. Cell Death Dis 5: e1084, 2014

5. Tian W, Xu D and Deng YC: Honokiol, a multifunctional tumor cell death inducer. Pharmazie 67: 811-816, 2012.

6. Li L, Han W, Gu Y, Qiu S, Lu Q, Jin J, Luo J and Hu X: Honokiol induces a necrotic cell death through the mitochondrial permeability transition pore. Cancer Res 67: 4894-4903, 2007.

7. Tian W, Deng Y, Li L, He H, Sun J and Xu D: Honokiol synergizes chemotherapy drugs in multidrug resistant breast cancer cells via enhanced apoptosis and additional programmed necrotic death Int J Oncol 42: 721-732, 2013.

8. Tian W, Xu D, Han W, He H, Cai H, Chen H, Zhou M, Chen J and Deng YC: Cyclophilin D modulates cell death transition from early apoptosis to programmed necrosis induced by honokiol. Int J Oncol 42: 1654-1663, 2013.

9. Remijsen Q, Goossens V, Grootjans S, Van den Haute C, Vanlangenakker N, Dondelinger Y, Roelandt R, Bruggeman I, Goncalves A, Bertrand MJ, et al: Depletion of RIPK3 or MLKL blocks TNF-driven necroptosis and switches towards a delayed RIPK1 kinase-dependent apoptosis. Cell Death Dis 5: e1004, 2014.

10. Shimobayashi M and Hall MN: Making new contacts: The mTOR network in metabolism and signalling crosstalk. Nat Rev Mol Cell Biol 15: 155-162, 2014.

11. Yang H, Rudge DG, Koos JD, Vaidialingam B, Yang HJ and Pavletich NP: mTOR kinase structure, mechanism and regulation. Nature 497: 217-223, 2013.

12. Beauchamp EM and Platanias LC: The evolution of the TOR pathway and its role in cancer. Oncogene 32: 3923-3932, 2013.

13. Hortobagyi GN, Chen D, Piccart M, Rugo HS, Burris HA III, Pritchard KI, Campone M, Noguchi S, Perez AT, Deleu I, et al: Correlative analysis of genetic alterations and everolimus benefit in hormone receptor-positive, human epidermal growth factor receptor 2-negative advanced breast cancer: Results from BOLERO-2. J Clin Oncol: 26 Oct 2015 (Epub ahead of print).

14. Linkermann A, Bräsen JH, Darding M, Jin MK, Sanz AB, Heller JO, De Zen F, Weinlich R, Ortiz A, Walczak H, et al: Two independent pathways of regulated necrosis mediate ischemia-reperfusion injury. Proc Natl Acad Sci USA 110: 12024-12029, 2013.
15. Baines CP, Kaiser RA, Purcell NH, Blair NS, Osinska H, Hambleton MA, Brunskill EW, Sayen MR, Gottlieb RA, Dorn GW, et al: Loss of cyclophilin D reveals a critical role for mitochondrial permeability transition in cell death. Nature 434: 658-662, 2005.

16. Kroemer G, Galluzzi L and Brenner C: Mitochondrial membrane permeabilization in cell death. Physiol Rev 87: 99-163, 2007.

17. Nakagawa T, Shimizu S, Watanabe T, Yamaguchi O, Otsu K, Yamagata H, Inohara H, Kubo T and Tsujimoto Y: Cyclophilin D-dependent mitochondrial permeability transition regulates some necrotic but not apoptotic cell death. Nature 434: 652-658, 2005.

18. Schinzel AC, Takeuchi O, Huang Z, Fisher JK, Zhou Z, Rubens J, Hetz C, Danial NN, Moskowitz MA and Korsmeyer SJ: Cyclophilin D is a component of mitochondrial permeability transition and mediates neuronal cell death after focal cerebral ischemia. Proc Natl Acad Sci USA 102: 12005-12010, 2005.

19. Wang H, Sun L, Su L, Rizo J, Liu L, Wang LF, Wang FS and Wang X: Mixed lineage kinase domain-like protein MLKL causes necrotic membrane disruption upon phosphorylation by RIP3. Mol Cell 54: 133-146, 2014.

20. Laplante M and Sabatini DM: mTOR signaling in growth control and disease. Cell 149: 274-293, 2012.

21. Zoncu R, Efeyan A and Sabatini DM: mTOR: From growth signal integration to cancer, diabetes and ageing. Nat Rev Mol Cell Biol 12: 21-35, 2011.

22. Park EJ, Min HY, Chung HJ, Hong JY, Kang YJ, Hung TM, Youn UJ, Kim YS, Bae K, Kang SS and Lee SK: Down-regulation of c-Src/EGFR-mediated signaling activation is involved in the honokiol-induced cell cycle arrest and apoptosis in MDA-MB-231 human breast cancer cells. Cancer Lett 277: 133-140, 2009.

23. Nagalingam A, Arbiser JL, Bonner MY, Saxena NK and Sharma D: Honokiol activates AMP-activated protein kinase in breast cancer cells via an LKB1-dependent pathway and inhibits breast carcinogenesis. Breast Cancer Res 14: R35, 2012.

24. Crane C, Panner A, Pieper RO, Arbiser J and Parsa AT: Honokiol-mediated inhibition of PI3K/mTOR pathway: A potential strategy to overcome immunoresistance in glioma, breast and prostate carcinoma without impacting $\mathrm{T}$ cell function. J Immunother 32: 585-592, 2009.

25. Kaushik G, Ramalingam S, Subramaniam D, Rangarajan P, Protti P, Rammamoorthy P, Anant S and Mammen JM: Honokiol induces cytotoxic and cytostatic effects in malignant melanoma cancer cells. Am J Surg 204: 868-873, 2012.

26. Nogueira V, Park Y, Chen CC, Xu PZ, Chen ML, Tonic I, Unterman $\mathrm{T}$ and Hay $\mathrm{N}$ : Akt determines replicative senescence and oxidative or oncogenic premature senescence and sensitizes cells to oxidative apoptosis. Cancer Cell 14: 458-470, 2008.

27. Kops GJ, Dansen TB, Polderman PE, Saarloos I, Wirtz KW, Coffer PJ, Huang TT, Bos JL, Medema RH and Burgering BM: Forkhead transcription factor FOXO3a protects quiescent cells from oxidative stress. Nature 419: 316-321, 2002. 